\title{
Pegylated liposomal doxorubicin: appraisal of its current role in the management of epithelial ovarian cancer
}

This article was published in the following Dove Press journal:

Cancer Management and Research

10 June 2011

Number of times this article has been viewed

\section{Maurie Markman}

Cancer Treatment Centers of America, Eastern Regional Medical Center, Philadelphia, PA, USA
Correspondence: Maurie Markman Cancer Treatment Centers of America, I33I East Wyoming Avenue, Philadelphia, PA 19124, USA

Tel +l 2155377502

Fax +l 2155373179

Email maurie.markman@ctca-hope.com
Abstract: Pegylated liposomal doxorubicin (PLD) has become a major component in the routine management of epithelial ovarian cancer. The drug is frequently employed as a single agent in the platinum-resistant setting, and recently reported data reveal the superiority of the combination of PLD plus carboplatin, compared with the platinum drug plus paclitaxel, in delaying the time to disease progression in women with recurrent (potentially platinum-sensitive) disease. Current research efforts involving PLD in ovarian cancer are focusing on adding novel targeted drugs to this cytotoxic agent. The utility of such approaches in the platinum-resistant population, compared with the sequential administration of single agents active in this setting, remains to be determined.

Keywords: PLD, carboplatin, paclitaxel, platinum-sensitive, platinum-resistant

\section{Standard management of epithelial ovarian cancer}

Initial management of epithelial ovarian cancer includes surgical staging and an attempt at optimal and, if possible, complete surgical cytoreduction. ${ }^{1}$

Surgery is followed by delivery of platinum-based chemotherapy, which in the setting of small volume residual disease may include the delivery of at least the platinum agent via the intraperitoneal route. ${ }^{1,2}$ Multiple trials conducted over the past decade have failed to document the superiority of a variety of approaches compared with the standard doublet of a platinum (carboplatin or cisplatin) agent plus a taxane (paclitaxel or docetaxel). ${ }^{3}$

An important Phase 3 trial conducted by the Japanese Gynecologic Oncology Group revealed the striking improvement in progression-free survival (PFS) associated with the weekly administration of paclitaxel compared with the standard every-3-week regimen, with an improvement in 3-year overall survival. ${ }^{4}$ Data regarding median overall survival are likely to be reported within the next year.

Two preliminary reports of Phase 3 trials that compared a carboplatin plus paclitaxel regimen to this two-drug program plus the addition of the anti-angiogenic agent, bevacizumab, revealed the novel program significantly improved PFS. ${ }^{5,6}$ Data on overall survival are pending.

An alternative approach to initial treatment of advanced ovarian cancer in the setting where the presence of extensive disease would appear to prevent successful initial surgical cytoreduction would be to deliver several cycles (generally 3 or 4) of platinum/taxane chemotherapy (after obtaining histological documentation of a malignancy consistent with an ovarian or primary peritoneal cancer) and to follow 
this with an attempt at resection of all macroscopic residual cancer. Recently reported Phase 3 trial data support this approach (neoadjuvant chemotherapy) to disease management in appropriately selected patients. ${ }^{7}$

In individuals who achieve a clinically defined complete response (normalization of serum CA-125 antigen level, normal CT [computed tomography] scan of the abdomen/pelvis, normal physical examination, no symptoms suggestive of persistent cancer), treatment is generally discontinued after the 6-8 platinum-based treatment cycles. One evidence-based trial revealed that the continuation of single agent monthly paclitaxel for a period of 12 months significantly delays the time to subsequent disease progression, ${ }^{8}$ but to date, there remains no evidence that this strategy (or any other maintenance approach) improves overall survival in epithelial ovarian cancer.

Unfortunately, despite the fact the majority of advanced ovarian cancer patients $(70 \%-80 \%)$ respond to platinumbased chemotherapy, ultimate recurrence of the disease is the rule, rather than the exception. ${ }^{1}$ A variety of management approaches may be utilized in this setting, with platinumbased treatment programs generally initially employed in women whose disease has remained in remission for at least 6 months (so-called, "platinum-sensitive recurrence"), although many in the gynecologic cancer research community believe it is only after a "platinum-free interval" of at least 12 months that it should be considered essentially required ("standard-of-care") to initially re-treat with a platinum-based program.

It is relevant to note here that there is no current evidence that any treatment regimen delivered to ovarian cancer patients whose disease had recurred following an initial response, or where the malignancy is documented to persist following the completion of 6-8 cycles of platinum-based primary chemotherapy, has the legitimate potential to cure the cancer, even if it is possible that survival may be prolonged with therapy known to be biologically and clinically active in this setting. Therefore, it is essential to appreciate that treatment in the "second-line" (and beyond) setting must be considered to be administered to improve disease-related symptoms, delay the time until the development of such symptoms, help optimize the patient's overall quality-oflife, and prolong overall survival, but not to permanently eliminate the malignancy.

In patients whose ovarian cancer recurs early, or where it is subsequently documented that the cancer is platinum resistant, a number of single agent anti-neoplastic drugs have been documented to produce objective response rates in more than $10 \%$ of the treated population (Table 1$).{ }^{9}$ Importantly, there is no current evidence-based Phase 3 trial data that any combination chemotherapy regimen is superior to single-agent treatment in platinum-resistant (platinumfree interval $<6$ months) ovarian cancer, nor that any one of multiple possible single agents that may be employed in this setting is therapeutically superior to alternative strategies. It should be noted, however, that the toxicity profiles of the individual agents that may be employed in this clinical circumstance vary substantially.

In general, the choice of treatment in the platinumresistant setting is made based on the previous toxicity experienced by the patient (eg, persistent neuropathy from prior platinum/taxane), the actual availability of, and payment for (eg, government, third-party private insurance) specific treatment options, and individual choice. Further, where available, treatment on a clinical trial may be a reasonable therapeutic option.

Currently, an individual ovarian cancer patient may ultimately receive as few as several and as many as a dozen treatment regimens during the natural history of her cancer. It is relevant to note here that the duration of survival following initial documentation of disease progression in ovarian cancer is increasingly likely to actually exceed the time period from diagnosis to the first evidence of recurrence.

Further, with the increased effectiveness of antineoplastic therapy in epithelial ovarian cancer, a substantial percentage of women presenting with advanced disease will be anticipated to survive for more than 5 years following initial diagnosis. As a result, it is not unreasonable to characterize this clinical scenario in many patients as being a very serious but also legitimate chronic disease process. ${ }^{10}$

Table I Anti-neoplastics with single-agent activity (objective responses $>10 \%$ ) in platinum-resistant ovarian cancer (platinumfree interval $<6$ months) (listed in alphabetical order)

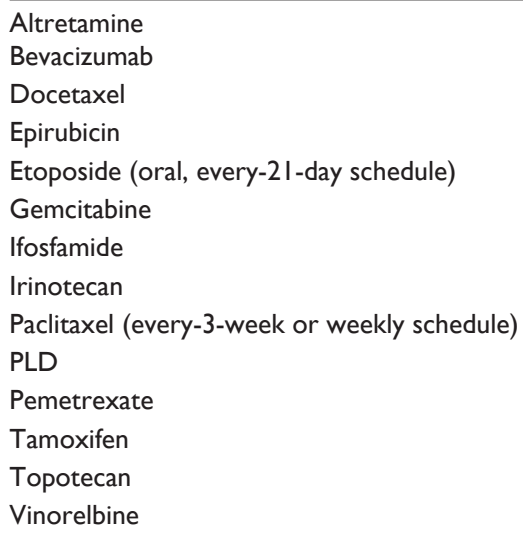

Abbreviation: PLD, pegylated liposomal doxorubicin. 


\section{Pegylated liposomal doxorubicin (PLD)}

PLD is a formulation of the anti-neoplastic agent, doxorubicin, encapsulated in a novel proprietary bilayer lipid sphere ("liposome"). ${ }^{11-14}$ This material is contained within and surrounded by a layer of polyethylene glycol. Pre-clinical evaluation revealed that the size (approximately $100 \mathrm{~nm}$ ) and chemical structure of this liposome selectively interfered with its entry into the vasculature of normal organs (heart, gastrointestinal tract, lungs, brain). ${ }^{11-14}$

In contrast, there was evidence for preferential deposition and prolonged exposure within tumor deposits whose blood vessels are often morphologically quite abnormal, permitting leakage into the surrounding tissue. In addition, the polyethylene glycol covering decreased protein binding and appears to increase the time of circulation within the systemic compartment.

\section{PLD in epithelial ovarian cancer: initial nonrandomized trial experience}

Phase 1 trials of PLD confirmed the extended exposure within the systemic compartment to doxorubicin following a single administration, with the toxicity profile of the agent being similar to that observed, with continuous infusion of the parent anti-neoplastic. ${ }^{15}$ This experience emphasized the importance of drug delivery on an every-4-week schedule, rather than a more frequent dosing strategy (eg, every 3 weeks) commonly used in oncology, including the standard use of doxorubicin itself.

In these early and subsequent clinical trials, as well as in routine clinical use, the dose-limiting side effects of PLD have included stomatitis, mucositis, and the development of palmar-plantar erythrodysesthesia (PPE), or the "hand-foot syndrome". ${ }^{16,17}$ Importantly, cardiac dysfunction appears to be far less of a concern with PLD than that associated with the parent drug (doxorubicin), and both complete alopecia and serious bone marrow suppression are less commonly observed.

Nonrandomized Phase 2 trials of PLD in platinumresistant ovarian cancer documented the biological activity of the agent in this clinical setting, with objective response rates of approximately $10 \%-20 \%$ being reported in several individual trials. ${ }^{16,18,19}$ It is relevant to note that this initial experience did not provide evidence that this agent was superior to alternative strategies that might have been employed in patients with platinum-resistant disease (eg, single agent topotecan), but in view of the limited therapeutic options in this difficult setting there was considerable interest to continue to explore the potential of this drug in ovarian cancer.

\section{PLD in epithelial ovarian cancer: single-agent randomized Phase 3 trial experience}

The initial randomized trial examining PLD in epithelial ovarian cancer compared this drug to single agent topotecan when employed as the initial "second-line" management strategy in women with both recurrent (platinum-free interval $>6$ months) and resistant (platinumfree interval $<6$ months) disease (Table 2). ${ }^{17,20}$ PLD was administered at a dose of $50 \mathrm{mg} / \mathrm{m}^{2}$ every 4 weeks and topotecan at $1.5 \mathrm{mg} / \mathrm{m}^{2} \times 5$ days every 3 weeks.

The results of this study, which have been widely discussed and debated in the literature, revealed no difference in either PFS or overall survival between the two strategies in the platinum-resistant setting. However, in patients with platinum-sensitive recurrent disease, there was a rather striking difference (in favor of the PLD arm) in overall survival, despite a far more modest improvement in PFS (in favor of PLD) (Table 2). ${ }^{17,20}$

While these results unequivocally support both the biological and clinical activity of PLD in ovarian cancer,

Table 2 Trials involving single agent PLD in epithelial ovarian cancer (median PFS and overall survival)

\begin{tabular}{|c|c|c|}
\hline Patient population & PLD & (Other agent) \\
\hline \multirow[t]{2}{*}{ Gordon et al $\left.\right|^{17,20}$} & & Topotecan \\
\hline & Dose/schedule: $50 \mathrm{mg} / \mathrm{m}^{2} \mathrm{q}-28 \mathrm{~d}$ & Dose/schedule: $1.5 \mathrm{~m} / \mathrm{m}^{2} \mathrm{q}-21 \mathrm{~d}$ \\
\hline Platinum-sensitive & PFS: 28.9 weeks; OS: 108 weeks & PFS: 23.3 weeks; OS: 7I.I weeks \\
\hline Platinum-resistant & PFS: 9.1 weeks; OS: 35.6 weeks & PFS: I 3.6 weeks; OS: 4 I.3 weeks \\
\hline Ferrandina et $\mathrm{a}^{25}$ & & Gemcitabine \\
\hline \multirow[t]{2}{*}{ Platinum-resistant } & Dose/schedule: $40 \mathrm{mg} / \mathrm{m}^{2} \mathrm{q}-28 \mathrm{~d}$ & Dose/schedule: $I, 000 \mathrm{mg} / \mathrm{m}^{2}$ day $\mathrm{I}, 8,15 \mathrm{q}-28 \mathrm{~d}$ \\
\hline & PFS: 16 weeks; OS: 56 weeks & PFS: 20 weeks; OS: 5 I weeks \\
\hline \multirow[t]{3}{*}{ Mutch et $\mathrm{a}^{24}$} & & Gemcitabine \\
\hline & Dose/schedule: $50 \mathrm{mg} / \mathrm{m}^{2} \mathrm{q}-28 \mathrm{~d}$ & Dose/schedule: $1000 \mathrm{mg} / \mathrm{m}^{2}$ day $\mathrm{I}, 8 \mathrm{q}-2 \mathrm{I} \mathrm{d}$ \\
\hline & PFS: 3.1 months; OS: I 3.5 months & PFS: 3.6 months; OS: 12.7 months \\
\hline
\end{tabular}

Abbreviations: OS, overall survival; PLD, pegylated liposomal doxorubicin; PFS, progression-free survival. 
appropriate interpretation of the trial results is difficult for several reasons. First, it has been noted that the comparison of single agent PLD to single agent topotecan in the recurrent ovarian cancer setting is actually rather irrelevant since the more rational comparison in this specific clinical circumstance would have been to a platinum drug (most likely carboplatin), either as a single agent or as combination strategy (see discussion of carboplatin/PLD versus carboplatin/paclitaxel Phase 3 trial below). This is due to the known major activity of platinum agents in this specific patient population. ${ }^{1,9,21,22}$

Second, it has been suggested that one likely reason for the greater impact of PLD on overall survival, compared with PFS, was that more patients randomized to the PLD study arm were subsequently able to receive a platinum agent following completion of treatment on this protocol compared with women randomized to the far more bone-marrow suppressing topotecan regimen (particularly when administered at the dose of $1.5 \mathrm{mg} / \mathrm{m}^{2} \times 5$ days). This interpretation, which remains entirely speculative as treatment employed following removal from this study was not routinely collected as part of the study design, concludes that the PLD study arm "won" largely because more patients randomized to this regimen were able to receive the single most active drug in the malignancy, a platinum agent.

A final frequently cited interpretation of the study results is that more patients who completed treatment on the PLD regimen were able to "cross-over" to the active agent, topotecan, than were able to receive PLD (at that time an "experimental drug" in this clinical setting) after they progressed on the topotecan study arm. Again, there are no data to directly support the validity of this claim as there is no evidence that the use of topotecan in this setting is either superior or even equivalent to a platinum agent, which would have been widely available to patients who completed treatment on this protocol.

Several additional Phase 3 trials have been reported which directly compared single agent PLD to other single agents (paclitaxel, gemcitabine) in platinum-resistant (platinumfree interval $<6$ months) and "modestly" platinum-sensitive (platinum-free interval 6-12 months) ovarian cancer (Table 2). ${ }^{23-25}$ While side-effect profiles of the agents often differed substantially, these studies essentially revealed the therapeutic equivalence for these agents in this difficult clinical setting.

Two additional points regarding the administration of single agent PLD in the second-line setting in women with epithelial ovarian cancer are worthy of highlighting before moving on to a discussion of evidence-based data evaluating PLD-containing combination chemotherapy regimens in this malignancy.

In an earlier section of this review it was noted that ovarian cancer patients frequently receive multiple lines of therapy following initial documentation of disease recurrence or persistence. However, the majority of evidencebased trials in ovarian cancer (and other malignancies) that explore "second-line treatment" strategies are actually only examining the first of many regimens used in this population. It is reasonable to inquire whether there is evidence that the administration of PLD, or other biologically active agents in ovarian cancer improves either PFS or overall survival when delivered in the "third-line" (or later) setting.

In fact, in a somewhat controversial trial, patients with advanced ovarian cancer who had progressed after two prior treatment regimens were randomized to a "control arm" of either topotecan or PLD (based on which drug they had not received as "second-line" treatment) or an "experimental drug" (canfosfamide) (Table 3). ${ }^{26}$ As patients treated on the study control arm experienced a highly statistically significant improvement in both PFS and overall survival compared with treatment with the "experimental regimen", these data provide relevant (although admittedly indirect) evidence that treatment with a known biologically and clinically active antineoplastic agent (including PLD and topotecan) as third-line treatment of ovarian cancer improves survival, compared with an inactive (or no) treatment in this setting.

The second point relates to the issue of the appropriate dose of single agent PLD to be used either as a "control arm" in ongoing and future clinical trials or in routine clinical practice. It was previously noted that the initial trials that examined the utility of PLD as second-line therapy of ovarian cancer employed a dose of $50 \mathrm{mg} / \mathrm{m}^{2}$ delivered on an every4-week schedule. ${ }^{17}$ This specific program was subsequently accepted for registration by drug regulatory authorities.

Unfortunately, the "50 mg/m² every-28-day"dose and schedule of PLD results in a substantial incidence (approximately 20\%-30\%) of grade 3 "hand-foot-syndrome". ${ }^{16,17}$ Considerable clinical experience generated since the

Table 3 PLD or topotecan versus canfosfamide as third-line chemotherapy of ovarian cancer ${ }^{26}$

\begin{tabular}{lll}
\hline Treatment & $\begin{array}{l}\text { Progression-free survival } \\
\text { (median) }\end{array}$ & $\begin{array}{l}\text { Overall survival } \\
\text { (median) }\end{array}$ \\
\hline PLD or topotecan & 4.3 months & 13.5 months \\
Canfosfamide & 2.3 months & 8.5 months \\
\hline
\end{tabular}

Note: $P<0.01$.

Abbreviation: PLD, pegylated liposomal doxorubicin. 
initial regulatory approval of PLD has revealed essentially equivalent clinical activity with substantially less severe adverse events when this agent is administered at a dose of $40 \mathrm{mg} / \mathrm{m}^{2}$ (rather than $50 \mathrm{mg} / \mathrm{m}^{2}$ ) on a 4-week schedule. ${ }^{19,27,28}$ Further, existing evidence reveals the large majority of ovarian cancer patients treated with PLD in this palliative setting outside the confines of a clinical trial will be given the lower $\left(40 \mathrm{mg} / \mathrm{m}^{2}\right)$ dose. ${ }^{29}$ Based on these considerations, there appears to be little (if any) ethical justification today for the administration of PLD at the initial regulatory agency-approved dose level.

In addition, the results of two randomized Phase 3 trials provide additional strong, but indirect support for the conclusion that the $40 \mathrm{mg} / \mathrm{m}^{2}$ dose level of PLD is therapeutically equivalent to the higher (and more toxic) dose approved for standard use in the second-line management of ovarian cancer (Table 2). ${ }^{24,25}$ In one study, single agent PLD, administered at a dose of $50 \mathrm{mg} / \mathrm{m}^{2}$ (every-4-week schedule) was compared with single agent gemcitabine, ${ }^{24}$ while in the second study, PLD was administered at a dose of $40 \mathrm{mg} / \mathrm{m}^{2}$ (every-4-week schedule) and again compared with gemcitabine. ${ }^{25}$ Both studies revealed essentially equivalent survival following treatment with single agent gemcitabine or either of the two PLD programs (40 or $50 \mathrm{mg} / \mathrm{m}^{2}$ ).

\section{PLD in epithelial ovarian cancer: randomized trials of combination chemotherapy regimens}

Several trials have explored the potential utility of PLD as a component of front-line treatment of advanced ovarian cancer. In a large multinational Phase 3 trial, one of four investigative study arms (compared with carboplatin plus paclitaxel) added PLD to the two-drug platinum/taxane combination. ${ }^{3}$ None of the investigational arms (including the addition of PLD) was shown to be superior to the standard carboplatin/paclitaxel regimen. ${ }^{3}$

In a recently reported and unfortunately underpowered Phase 3 trial, investigators substituted PLD for paclitaxel in the front-line chemotherapy program and compared this approach to the standard platinum/taxane regimen (Table 4). ${ }^{30}$

Table 4 Carboplatin/PLD versus carboplatin/paclitaxel as initial chemotherapy of ovarian cancer ${ }^{30}$

\begin{tabular}{lll}
\hline Treatment & $\begin{array}{l}\text { Progression-free survival } \\
\text { (median) }^{\mathbf{a}}\end{array}$ & $\begin{array}{l}\text { Overall survival } \\
\text { (median) }^{\mathbf{b}}\end{array}$ \\
\hline Carboplatin/PLD & 19.0 months & 61.6 months \\
Carboplatin/paclitaxel & 16.8 months & 53.2 months \\
\hline
\end{tabular}

Notes: ${ }^{\mathrm{a}} \mathrm{P}=0.58 ;{ }^{\mathrm{b}} \mathrm{P}=0.32$.

Abbreviation: PLD, pegylated liposomal doxorubicin.
While there were no statistically significant differences in either PFS or overall survival between the treatment arms, the trial was actually designed as a superiority (rather than a noninferiority) study. As a result, the appropriate interpretation is that the carboplatin/PLD arm failed to demonstrate superiority to standard carboplatin/paclitaxel, and not that the two study regimens were equivalent in efficacy.

In contrast to the difficult-to-interpret carboplatin/PLD front-line ovarian cancer study, a Phase 3 trial that directly compared carboplatin/PLD with carboplatin/paclitaxel as treatment of platinum-sensitive recurrent disease revealed rather surprising superiority for the PLD-containing program in PFS (median 11.3 months versus 9.3 months, $P=0.005$ ) (Table 5). ${ }^{31}$ At the time of the report of this study, there was no difference in overall survival between the two study arms.

Another unexpected, currently largely unexplained, but potentially highly clinically relevant difference between the regimens in this "second-line" study was a statistically significantly reduced incidence in carboplatin-associated hypersensitivity reactions observed following treatment with the PLD-containing program, compared with the combination containing paclitaxel. ${ }^{30}$ The lower incidence of this clinically relevant side effect might have translated to a measurably greater percentage of patients who were able to receive a sufficient number of carboplatin treatment cycles to optimize their chances to experience benefit from the agent recognized to be the most important cytotoxic drug in the management of this malignancy. $1,21,22$

Of interest, a second much smaller randomized trial that compared single agent carboplatin to the combination of carboplatin plus PLD revealed a similar strikingly lower incidence of carboplatin-associated hypersensitivity in patients randomized to the PLD-containing program (Table 5). ${ }^{32}$

Another Phase 3 randomized trial compared the combination of PLD plus trabectedin versus PLD alone in both platinum-resistant and "moderately" platinum-sensitive (platinum-free interval 6-12 months) recurrent ovarian cancer. ${ }^{33}$ As with the previously discussed Phase 3 trials in platinum-resistant ovarian cancer, there was no difference in PFS between the two study arms in this setting, but side effects were greater with the combination strategy (Table 6).

However, in the "moderately" platinum-sensitive population the two-drug program was associated with a statistically significant improvement in PFS. Data on overall survival remained immature at the time of the initial study report. Unfortunately, the improvement in PFS, while potentially highly clinically relevant was accompanied by a greater 
Table 5 Carboplatin/PLD versus carboplatin/paclitaxel or single agent carboplatin as second-line chemotherapy of ovarian cancer

\begin{tabular}{|c|c|c|c|c|}
\hline Study & Treatment & $\begin{array}{l}\text { Progression-free } \\
\text { survival (median) }\end{array}$ & $\begin{array}{l}\text { Overall survival } \\
\text { (median) }\end{array}$ & $\begin{array}{l}\text { Allergic/hypersensitivity reactions } \\
\text { (\% of patients developing) }\end{array}$ \\
\hline \multirow[t]{3}{*}{ Pujade-Lauraine et $\mathrm{a}^{31}$} & Carboplatin/PLD & 11.3 months & (too early to report) & $5.6 \%$ \\
\hline & Carboplatin/Paclitaxel & 9.4 months & & $18.8 \%$ \\
\hline & & $(P=0.005)$ & & \\
\hline \multirow[t]{3}{*}{ Markman et a ${ }^{32}$} & Carboplatin/PLD & 12 months & 31 months & $0 \%$ (0 of 3 I patients) \\
\hline & Carboplatin (single agent) & 8 months & 18 months & $30 \%$ ( 9 of 30 patients) \\
\hline & & $(P=0.02)$ & $(P=0.2)$ & \\
\hline
\end{tabular}

Abbreviation: PLD, pegylated liposomal doxorubicin.

incidence of serious toxicity. As a result, it is reasonable to inquire if this statistically significant improvement in time to disease progression actually translates into meaningful clinical benefit.

Further, similar to the previous discussion regarding interpretation of the recurrent disease data in the single agent PLD versus single agent topotecan clinical trial, it remains unknown how the two drug combination of PLD/ trabectadin would compare with single agent carboplatin, carboplatin plus PLD, or alternative carboplatin-based combination chemotherapy strategies (eg, with gemcitabine) in this clinical setting.

\section{PLD in epithelial ovarian cancer: future directions}

Early phase trials have begun to explore the potential of adding PLD in combination with a variety of alternative drugs, including bevacizumab, and other "targeted agents" in management of epithelial ovarian cancer.

With the demonstrated superiority of carboplatin/PLD compared with carboplatin/paclitaxel in recurrent ovarian cancer, ${ }^{31}$ and the documented favorable impact on PFS associated with the addition of bevacizumab to front-line cytotoxic chemotherapy, ${ }^{5,6}$ it will be quite relevant to explore the addition of this anti-angiogenic agent with the PLD-containing carboplatin combination in the second-line setting. Such a

Table 6 PLD/trabectedin versus single agent PLD as second-line chemotherapy of ovarian cancer $^{33}$

\begin{tabular}{lll}
\hline & PLD & PLD/trabectedin \\
\hline $\begin{array}{l}\text { Platinum-resistant } \\
\text { Progression-free survival (median) }\end{array}$ & 3.7 months & $\begin{array}{l}4.0 \text { months } \\
(P=0.75)\end{array}$ \\
$\begin{array}{l}\text { “Modestly" platinum-sensitive } \\
\text { Progression-free survival (median) }\end{array}$ & 7.5 months & $\begin{array}{l}9.2 \text { months } \\
(P=0.017)\end{array}$ \\
$\begin{array}{l}\text { Total patient population } \\
\text { Progression-free survival (median) }\end{array}$ & 5.8 months & $\begin{array}{l}7.3 \text { months } \\
(P=0.019)\end{array}$ \\
\hline
\end{tabular}

Abbreviation: PLD, pegylated liposomal doxorubicin. trial will be of particular interest if the pending results of a Phase 3 trial that compares the combination of carboplatin plus gemcitabine with carbopatin/gemcitabine plus bevacizumab in recurrent ovarian cancer reveals the superiority of the three-drug approach.

As bevacizumab, and other anti-angiogenic agents, are known to potentially impair wound healing, it may be particularly relevant to make major efforts to avoid PLDinduced mucous membrane or skin toxicity when this drug is combined with an anti-angiogenic. Data from the clinical trials experience will hopefully reveal the toxicity profile of such combinations, but caution is advised until such safety information is available.

\section{Disclosure}

The author reports no conflicts of interest in this work.

\section{References}

1. Hennessy BT, Coleman RL, Markman M. Ovarian cancer. Lancet. 2009;374:1371-1382.

2. Markman M, Walker JL. Intraperitoneal chemotherapy of ovarian cancer: a review with a focus on practical aspects of treatment. J Clin Oncol. 2006;24:988-994.

3. Bookman MA, Brady MF, McGuire WP, et al. Evaluation of new platinum-based treatment regimens in advanced-stage ovarian cancer: a Phase III trial of the Gynecologic Cancer Intergroup. J Clin Oncol. 2009;27:1419-1425.

4. Katsumata N, Yasuda M, Takahashi F, et al. Dose-dense paclitaxel once a week in combination with carboplatin every 3 weeks for advanced ovarian cancer: a phase 3, open-label, randomized controlled trial. Lancet. 2009;374:1331-1338.

5. Burger RA, Bookman MA, Walker JL, et al. Phase III trial of bevacizumab in the primary treatment of advanced epithelial ovarian cancer, primary peritoneal cancer, or fallopian tube cancer: a Gynecologic Oncology Group study. J Clin Oncol. 2010;28(18s):LBA1.

6. Perren T, Swart AM, Pfisterer J, et al. ICON 7: a Phase III Gynecologic Cancer Intergroup (GCIG) trial of adding bevacizumab to standard chemotherapy in women with newly diagnosed epithelial ovarian, primary peritoneal or fallopian tube cancer. Presentation at ESMO, Milan, Italy; 2010.

7. Vergote I, Trope CG, Amant F, et al. Neoadjuvant chemotherapy or primary surgery in stage IIIC or IV ovarian cancer. $N$ Engl J Med. 2010; 363:943-953.

8. Markman M, Liu PY, Wilczynski S, et al. Phase III randomized trial of 12 versus 3 months of maintenance paclitaxel in patients with advanced ovarian cancer after complete response to platinum-based chemotherapy: a Southwest Oncology Group and Gynecologic Oncology Group trial. J Clin Oncol. 2003;21:2460-2465. 
9. Markman M, Bookman M. Second-line treatment of ovarian cancer. Oncologist. 2000;6:26-35.

10. Markman M. Viewing ovarian cancer as a "chronic disease": what exactly does this mean? Gynecol Oncol. 2006;100:229-230.

11. Papahadjopoulous D, Allen TN, Gabizon A, et al. Sterically stabilized liposomes: Improvement in pharmacokinetic and antitumor therapeutic efficacy. Proc Natl Acad Sci U S A. 1991;88:11460-11464.

12. Vaage J, Donovan D, Mayhew E, et al. Therapy of human ovarian carcinoma xenographs using doxorubicin encapsulated in sterically stabilized liposomes. Cancer. 1993;72:3671-3675.

13. Vaage J, Barbert-Guillem E, Abra R, et al. Tissue distribution and therapeutic effects of intravenous free and liposomal doxorubicin against prostatic carcinoma xenographs. Cancer. 1994;73:1478-1484.

14. Huang SK, Mayhew E, Gilani S. et al. Pharmacokinetics and therapeutics of sterically stabilized liposomes in mice bearing C-26 colon carcinoma. Cancer Res. 1992;52:6774-6781.

15. Uziely B, Jeffers S, Isacson R, et al. Liposomal doxorubicin (Dox-SL ${ }^{\mathrm{TM}}$ ): antitumor activity and unique toxicities during two complementary Phase I studies. J Clin Oncol. 1995;13:1777-1785.

16. Muggia FM, Hainsworth JD, Jeffers S, et al. Phase II study of liposomal doxorubicin in refractory ovarian cancer: Antitumor activity and toxicity modification by liposomal encapsulation. J Clin Oncol. 1997;15: 987-993.

17. Gordon AN, Fleagle JT, Guthrie D, et al. Recurrent epithelial ovarian carcinoma: a randomized phase III study of pegylated liposomal doxorubicin versus topotecan. J Clin Oncol. 2001;19:3312-3322.

18. Gordon AN, Granai CO, Rose PG, et al. Phase II study of liposomal doxorubicin in platinum- and paclitaxel-refractory epithelial ovarian cancer. J Clin Oncol. 2000;18:3093-3100.

19. Markman M, Kennedy A, Webster K, et al. Phase 2 trial of liposomal doxorubicin $\left(40 \mathrm{mg} / \mathrm{m}^{2}\right)$ in platinum/paclitaxel-refractory ovarian and fallopian tube cancers and primary carcinoma of the peritoneum. Gynecol Oncol. 2000;78:369-372.

20. Gordon AN, Tonda M, Sun S, et al. Long-term survival advantage for women treated with pegylated liposomal doxorubicin compared with topotecan in a Phase 3 randomized study of recurrent and refractory epithelial ovarian cancer. Gynecol Oncol. 2004;95:1-8.

21. Muggia FM, Braly PS, Brady MF, et al. Phase III randomized study of cisplatin versus paclitaxel versus cisplatin and paclitaxel in patients with suboptimal stage III or IV ovarian cancer: a Gynecologic Oncology Group study. J Clin Oncol. 2000;18:106-115.
22. Gore ME, Fryatt I, Wiltshaw E, et al. Treatment of relapsed carcinoma of the ovary with cisplatin or carboplatin following initial treatment with these compounds. Gynecol Oncol. 1990;13:383-393.

23. O'Byrne KS, Bliss P, Graham JD, et al. A Phase III study of Doxil/ Caelyx versus paclitaxel in platinum-treated, taxane-naïve relapsed ovarian cancer. Proc Am Soc Clin Oncol. 2002;21:203a.

24. Mutch DG, Orlando M, Goss T, et al. Randomized phase III trial of gemcitabine compared with pegylated liposomal doxorubicin in patients with platinum-resistant ovarian cancer. J Clin Oncol 2007;25:2811-2818.

25. Ferrandina G, Ludovisi M, Lorusso D, et al. Phase III trial of gemcitabine compared with pegylated liposomal doxorubicin in progressive or recurrent ovarian cancer. J Clin Oncol. 2008;26:890-896.

26. Vergote I, Finkler N, del Campo J, et al. Phase 3 randomized study of canfosfamide (Telcyda ${ }^{\circledR}$, TLK286) versus pegylated liposomal doxorubicin or topotecan as third-line therapy in patients with platinum-refractory or -resistant ovarian cancer. Eur J Cancer. 2009;45: 2324-2332.

27. Rose PG, Maxson JH, Fusco N, et al. Liposomal doxorubicin in ovarian, peritoneal, and tubal carcinoma: a retrospective comparative study of single-agent dosages. Gynecol Oncol. 2001;82:323-328.

28. Campos SM, Penson RT, Mays AR, et al. The clinical utility of liposomal doxorubicin in recurrent ovarian cancer. Gynecol Oncol. 2001;81: 206-212.

29. Tanyi JL, Smith JA, Ramos L, et al. Predisposing risk factors for palmar-plantar erythrodysesthesia when using liposomal doxorubicin to treat recurrent ovarian cancer. Gynecol Oncol. 2009;114:219-224.

30. Pignata S, Scambia G, Savarese A, et al. Carboplatin plus paclitaxel versus carboplatin plus pegylated liposomal doxorubicin in patients with advanced ovarian cancer: final analysis of the MITO-2 randomized multicenter trial. J Clin Oncol. 2010;28(18s):952a.

31. Pujade-Lauraine E, Wagner U, Aavall-Lundqvist E, et al. Pegylated liposomal doxorubicin and carboplatin compared with paclitaxel and carboplatin for patients with platinum-sensitive ovarian cancer in late relapse. J Clin Oncol. 2010;28:3323-3329.

32. Markman M, Moon J, Wilczynski S, et al. Single agent carboplatin versus carboplatin plus pegylated liposomal doxorubicin in recurrent ovarian cancer: final survival results of a SWOG (S0200) Phase 3 randomized trial. Gynecol Oncol. 2010;116:323-325.

33. Monk BJ, Herzog TJ, Kaye SB, et al. Trabectedin plus pegylated liposomal doxorubicin in recurrent ovarian cancer. J Clin Oncol. 2010;28: 3107-3114.
Cancer Management and Research

\section{Publish your work in this journal}

Cancer Management and Research is an international, peer-reviewed open access journal focusing on cancer research and the optimal use of preventative and integrated treatment interventions to achieve improved outcomes, enhanced survival and quality of life for the cancer patient The journal welcomes original research, clinical \& epidemiological

\section{Dovepress}

studies, reviews \& evaluations, guidelines, expert opinion \& commentary, case reports \& extended reports. The manuscript management system is completely online and includes a very quick and fair peerreview system, which is all easy to use. Visit http://www.dovepress.com/ testimonials.php to read real quotes from published authors. 